\title{
Strong solutions of semilinear stochastic partial differential equations
}

\author{
Martina Hofmanová
}

\begin{abstract}
We study the Cauchy problem for a semilinear stochastic partial differential equation driven by a finite-dimensional Wiener process. In particular, under the hypothesis that all the coefficients are sufficiently smooth and have bounded derivatives, we consider the equation in the context of power scale generated by a strongly elliptic differential operator. Application of semigroup arguments then yields the existence of a continuous strong solution.
\end{abstract}

Mathematics Subject Classification (2000). 60H15, 35R60.

Keywords. Stochastic partial differential equations, Strongly elliptic differential operator, Strongly continuous semigroup.

\section{Introduction}

In the present paper, we consider the following semilinear stochastic partial differential equation driven by a finite-dimensional Wiener process:

$$
\begin{aligned}
\mathrm{d} u & =[\mathcal{A} u+F(u)] \mathrm{d} t+\sigma(u) \mathrm{d} W, \quad x \in \mathbb{T}^{N}, t \in(0, T), \\
u(0) & =u_{0},
\end{aligned}
$$

where $-\mathcal{A}$ is a strongly elliptic differential operator, $F$ is generally nonlinear unbounded operator and the diffusion coefficient in the stochastic term is also nonlinear.

It is a well known fact in the field of PDEs and SPDEs that many equations do not, in general, have classical or strong solutions and can be solved only in some weaker sense. Unlike deterministic problems, in the case of stochastic equations we can only ask whether the solution is smooth in the space variable. Thus, the aim of the present work is to determine conditions on coefficients and initial data under which there exists a spatially smooth solution to 
(1.1). The motivation for such a regularity result came from our research in the field of degenerate parabolic SPDEs of second order (see [10]), where smooth solutions of certain approximate nondegenerate problems were needed in order to derive the so-called kinetic formulation and to obtain kinetic solution. Nevertheless, since the regularity result of the present paper is based on properties of strongly elliptic operators, generalization to higher order equations does not cause any additional problems.

The literature devoted to the existence of a classical solution to deterministic parabolic problems is quite extensive, let us mention for instance the works of Friedman [7], Grunau and von Wahl [8], Ladyzhenskaya et al. [14], Lieberman [15], von Wahl [19], Yagi [20] and the references therein. Regularity in the case of linear parabolic SPDEs was treated by Krylov [11], Krylov and Rozovskii $[12,13]$ and the references therein, and Flandoli [6]. However, there seems to be less papers concentrated on regularity for nonlinear SPDEs. The starting point for our research was the paper of Gyöngy and Rovira [9] who studied a class of second order parabolic semilinear SPDEs. However, they were only concerned with $L^{p}$-valued solutions so our work can be regarded as an extension of their result. Related problems were also discussed by Zhang $[21,22]$, nevertheless, his assumptions are not satisfied in our case.

The main difficulty in the case of semilinear equations lies in the nonlinearities $F$ and $\sigma$ as, in higher order Sobolev spaces, we cannot expect the Lipschitz condition to be satisfied and hence the fixed point argument cannot be applied. This issue is closely related to the mapping properties of Nemytskij operators, i.e. $T_{G}: h \mapsto G(h)$, where $h$ belongs to some function space $E$ and $G: \mathbb{R} \rightarrow \mathbb{R}$ is nonlinear. It turns out (and was discussed in-depth in the book of Runst and Sickel [18]) that the mapping properties of these operators depend strongly on the chosen domain of definition and even for $E$ being a Sobolev space they do not, in general, map $E$ to itself.

Let us make things clearer on a simple example of a heat equation with a nonlinear right-hand side

$$
\partial_{t} u=\Delta u+H(u), \quad x \in \mathbb{T}^{N}, t \in(0, T) .
$$

Let $p \in[1, \infty)$. If $H: \mathbb{R} \rightarrow \mathbb{R}$ is Lipschitz continuous then

$$
\left\|H\left(z_{1}\right)-H\left(z_{2}\right)\right\|_{L^{p}\left(\mathbb{T}^{N}\right)} \leq C\left\|z_{1}-z_{2}\right\|_{L^{p}\left(\mathbb{T}^{N}\right)}, \quad z_{1}, z_{2} \in L^{p}\left(\mathbb{T}^{N}\right),
$$

therefore, as an easy consequence of the Banach fixed point theorem, there exists a unique mild solution to (1.2) in $L^{p}\left(\mathbb{T}^{N}\right)$. However, if $m \geq 1$ it is not generally true that

$$
\left\|H\left(z_{1}\right)-H\left(z_{2}\right)\right\|_{W^{m, p}\left(\mathbb{T}^{N}\right)} \leq C\left\|z_{1}-z_{2}\right\|_{W^{m, p}\left(\mathbb{T}^{N}\right)}, \quad z_{1}, z_{2} \in W^{m, p}\left(\mathbb{T}^{N}\right),
$$

so the existence of a solution in higher order Sobolev spaces cannot be proved directly. In fact, even the linear growth condition fails for $m \geq 2$ since the norm of a superposition does not grow linearly with the norm of the inner function. For example, if we consider $2 \leq m \leq N / p, p \in(1, \infty)$, then only linear operators map $W^{m, p}\left(\mathbb{T}^{N}\right)$ to itself (see [18, Theorem 5.2.4/2]).

On the other hand, for any $m \in \mathbb{N}$ and $p \in[1, \infty)$, under the hypothesis of a sufficiently smooth function $H$ having bounded derivatives one arrives at the 
fact that the Nemytskij operator $T_{H}$ maps $W^{1, m p}\left(\mathbb{T}^{N}\right) \cap W^{m, p}\left(\mathbb{T}^{N}\right)$ to itself and the following estimate holds true for any $z \in W^{m, p}\left(\mathbb{T}^{N}\right) \cap W^{1, m p}\left(\mathbb{T}^{N}\right)$ (cf. Proposition 3.1, Corollary 3.2 and Remark 3.3)

$$
\|H(z)\|_{W^{m, p}\left(\mathbb{T}^{N}\right)} \leq C\left(1+\|z\|_{W^{m, p}\left(\mathbb{T}^{N}\right)}+\|z\|_{W^{1, m p}\left(\mathbb{T}^{N}\right)}^{m}\right) .
$$

It turns out to be the keystone of our proof of regularity. In particular, we proceed successively in several steps. First of all, we consider the equation (1.2) in $L^{m p}\left(\mathbb{T}^{N}\right)$ and apply the Banach fixed point theorem to conclude the existence of an $L^{m p}\left(\mathbb{T}^{N}\right)$-valued mild solution. Next, we study its Picard iterations as processes having values in the Sobolev spaces $W^{1, m p}\left(\mathbb{T}^{N}\right)$. Having known that $T_{H}$ maps $W^{1, m p}\left(\mathbb{T}^{N}\right)$ to itself we are able to find a uniform estimate of the $W^{1, m p}\left(\mathbb{T}^{N}\right)$-norm which is then used in the last step to deduce a uniform estimate of the $W^{m, p}\left(\mathbb{T}^{N}\right)$-norm. Both estimates remain valid also for the limit process and, as a consequence, the mild solution to (1.2) is even strong (for a detailed exposition of these two concepts of solution we refer the reader to $[5])$.

Unlike the introduction, in the proof of the main result, Theorem 2.1, the integrability exponent $p$ is only allowed to take values in $[2, \infty)$ which is given by the use of the stochastic Itô integration in 2-smooth Banach spaces $($ see $[4,16])$.

As an immediate consequence of the main result, we obtain a continuous $C^{k, \lambda}$-valued solution. Here, we use the Sobolev embedding theorem so the stochastic integration in Banach spaces, i.e. $W^{m, p}$, allows us to weaken the smoothness assumptions on coefficients. We note that the regularity of the solution depends only on the regularity of the coefficients and the initial data and is not limited by the order of the equation.

The paper is organized as follows. In Sect. 2, we review the basic setting and state our main result. In Sect. 3, we collect important preliminary results related to Nemytskij operators. In the final section, these results are applied and the proof of the main theorem is established.

\section{Setting and the main result}

Let us first introduce the notation which will be used later on. We will consider periodic boundary conditions: $x \in \mathbb{T}^{N}$ where $\mathbb{T}^{N}$ is the $N$-dimensional torus. The Sobolev spaces on $\mathbb{T}^{N}$ will be denoted by $W^{m, p}\left(\mathbb{T}^{N}\right)$ and by $W^{m, p}\left(\mathbb{T}^{N} ; \mathbb{R}^{n}\right)$ we will denote the space of all functions $z=\left(z_{1}, \ldots, z_{n}\right): \mathbb{T}^{N} \rightarrow \mathbb{R}^{n}$ such that $z_{i} \in W^{m, p}\left(\mathbb{T}^{N}\right), i=1, \ldots, n$.

We now give the precise assumptions on each of the terms appearing in the above equation (1.1). We will work on a finite-time interval $[0, T], T>0$. The operator $-\mathcal{A}$ is a strongly elliptic differential operator of order $2 l$ with variable coefficients of class $C^{\infty}\left(\mathbb{T}^{N}\right)$. Let us assume, in addition, that $-\mathcal{A}$ is formally symmetric and positive, i.e. we assume that 0 belongs to the resolvent 
set of $-\mathcal{A}$. As an example of this operator let us mention for instance the second order differential operator in divergence form given by

$$
\mathcal{A} u=\sum_{i, j=1}^{N} \partial_{x_{i}}\left(A_{i j}(x) \partial_{x_{j}} u\right)
$$

where the coefficients $A_{i j}=A_{j i}$ are real-valued smooth functions and satisfy the uniform ellipticity condition, i.e. there exists $\alpha>0$ such that

$$
\sum_{i, j=1}^{N} A_{i j}(x) \zeta_{i} \zeta_{j} \geq \alpha|\zeta|^{2}, \quad \forall x \in \mathbb{T}^{N}, \quad \forall \zeta \in \mathbb{R}^{N}
$$

Let us now collect basic facts concerning strongly elliptic differential operators satisfying our hypotheses (for a detailed exposition we refer the reader to [17]). Set $D\left(\mathcal{A}_{p}\right)=W^{2 l, p}\left(\mathbb{T}^{N}\right)$. Then the linear unbounded operator $\mathcal{A}_{p}$ in $L^{p}\left(\mathbb{T}^{N}\right)$ defined by

$$
\mathcal{A}_{p} u=\mathcal{A} u, \quad u \in D\left(\mathcal{A}_{p}\right),
$$

is the infinitesimal generator of a bounded analytic semigroup on $L^{p}\left(\mathbb{T}^{N}\right)$. Let us denote this semigroup by $\mathcal{S}_{p}$. Fractional powers of $-\mathcal{A}_{p}$ are well defined and their domains correspond to classical Sobolev spaces (see [1, Section 10]), i.e.

$$
\left(D\left(\left(-\mathcal{A}_{p}\right)^{\delta}\right),\left\|\left(-\mathcal{A}_{p}\right)^{\delta} \cdot\right\|_{L^{p}\left(\mathbb{T}^{N}\right)}\right) \cong\left(W^{2 l \delta, p}\left(\mathbb{T}^{N}\right),\|\cdot\|_{W^{2 l \delta, p}\left(\mathbb{T}^{N}\right)}\right), \quad \delta \geq 0 .
$$

We will also make use of the following property of analytic semigroups (see [17, Chapter 2, Theorem 6.13]):

$\forall t>0 \quad \forall \delta>0$ the operator $\left(-\mathcal{A}_{p}\right)^{\delta} \mathcal{S}_{p}(t)$ is bounded in $L^{p}\left(\mathbb{T}^{N}\right)$,

$$
\left\|\left(-\mathcal{A}_{p}\right)^{\delta} \mathcal{S}_{p}(t)\right\| \leq C_{\delta, p} t^{-\delta}
$$

(here $\|\cdot\|$ stands for the operator norm).

The nonlinear term $F$ is defined as follows: for any $p \in[2, \infty)$

$$
\begin{aligned}
F: L^{p}\left(\mathbb{T}^{N}\right) & \longrightarrow W^{-2 l+1, p}\left(\mathbb{T}^{N}\right) \\
z & \longmapsto \sum_{|\alpha| \leq 2 l-1} a_{\alpha} \mathrm{D}^{\alpha} f_{\alpha}(z),
\end{aligned}
$$

where $a_{\alpha} \in \mathbb{R}$ and the functions $f_{\alpha},|\alpha| \leq 2 l-1$, are smooth enough (exact assumptions will be given later). Let us denote by $f$ the vector of functions $\left(f_{\alpha} ;|\alpha| \leq 2 l-1, a_{\alpha} \neq 0\right)$ and denote its length by $\eta$.

Throughout this article we fix a stochastic basis $\left(\Omega, \mathscr{F},\left(\mathscr{F}_{t}\right)_{t \geq 0}, \mathbb{P}\right)$ with a complete, right-continuous filtration. Let $\mathcal{P}$ denote the predictable $\sigma$-algebra on $\Omega \times[0, T]$ associated with $\left(\mathscr{F}_{t}\right)_{t \geq 0}$. For simplicity we will only consider finite-dimensional noise, however, the result can be extended to the infinitedimensional case. Let $\mathfrak{U}$ be a finite-dimensional Hilbert space and let $\left\{e_{i}\right\}_{i=1}^{d}$ be its orthonormal basis. The process $W$ is a $d$-dimensional $\left(\mathscr{F}_{t}\right)$-Wiener process in $\mathfrak{U}$, i.e. it has an expansion of the form $W(t)=\sum_{i=1}^{d} W_{i}(t) e_{i}$, 
where $W_{i}, i=1, \ldots, d$, are mutually independent real-valued standard Wiener processes relative to $\left(\mathscr{F}_{t}\right)_{t \geq 0}$. The diffusion coefficient $\sigma$ is then defined as

$$
\begin{aligned}
\sigma(z): & \mathfrak{U} \longrightarrow L^{p}\left(\mathbb{T}^{N}\right) \\
h & \longmapsto \sum_{i=1}^{d} \sigma_{i}(\cdot, z(\cdot))\left\langle e_{i}, h\right\rangle, \quad z \in L^{p}\left(\mathbb{T}^{N}\right),
\end{aligned}
$$

where the functions $\sigma_{1}, \ldots, \sigma_{d}: \mathbb{T}^{N} \times \mathbb{R} \rightarrow \mathbb{R}$ satisfy the following linear growth condition

$$
\sum_{i=1}^{d}\left|\sigma_{i}(x, \xi)\right|^{2} \leq C\left(1+|\xi|^{2}\right), \quad x \in \mathbb{T}^{N}, \xi \in \mathbb{R} .
$$

Since we are going to solve (1.1) in $L^{p}\left(\mathbb{T}^{N}\right)$, for $p \in[2, \infty)$, we need to ensure the existence of the stochastic integral as an $L^{p}\left(\mathbb{T}^{N}\right)$-valued process. Recall, that $L^{p}$ spaces, $p \in[2, \infty)$, as well as the Sobolev spaces $W^{m, p}, p \in$ $[2, \infty), m \geq 0$, belong to a class of the so-called 2-smooth Banach spaces, which are well suited for stochastic Itô integration. (A detailed construction of stochastic integral for processes with values in 2-smooth Banach spaces can be found in [4] or [16].) Let us denote by $\gamma(\mathfrak{U} ; X)$ the space of all $\gamma$ radonifying operators from $\mathfrak{U}$ to a 2 -smooth Banach space $X$. We will show that $\sigma(z) \in \gamma\left(\mathfrak{U} ; L^{p}\left(\mathbb{T}^{N}\right)\right)$ for any $z \in L^{p}\left(\mathbb{T}^{N}\right)$ and

$$
\|\sigma(z)\|_{\gamma\left(\mathfrak{U} ; L^{p}\left(\mathbb{T}^{N}\right)\right)}^{2} \leq C\left(1+\|z\|_{L^{p}\left(\mathbb{T}^{N}\right)}^{2}\right) .
$$

Note, that the following fact holds true:

$\forall s>0 \quad \exists C_{s} \in(0, \infty) \quad \forall \gamma_{1}, \ldots, \gamma_{d}$ independent $\mathcal{N}(0,1)$-random variables

$$
\forall r_{1}, \ldots, r_{d} \in \mathbb{R} \quad\left(\mathbb{E}\left|\sum_{i=1}^{d} r_{i} \gamma_{i}\right|^{s}\right)^{\frac{1}{s}}=C_{s}\left(\sum_{i=1}^{d} r_{i}^{2}\right)^{\frac{1}{2}} .
$$

The proof is, by the way, easy: $\left(\sum_{i=1}^{d} r_{i}^{2}\right)^{-\frac{1}{2}} \sum_{i=1}^{d} r_{i} \gamma_{i}$ is an $\mathcal{N}(0,1)$-random variable. Let $\left\{\gamma_{i}\right\}_{i=1}^{d}$ be a sequence of independent $\mathcal{N}(0,1)$-random variables, by the definition of a $\gamma$-radonifying norm, using (2.3) and (2.2)

$$
\begin{aligned}
& \|\sigma(z)\|_{\gamma\left(\mathfrak{U} ; L^{p}\left(\mathbb{T}^{N}\right)\right)}^{2}=\mathbb{E}\left\|\sum_{i=1}^{d} \gamma_{i} \sigma(z) e_{i}\right\|_{L^{p}\left(\mathbb{T}^{N}\right)}^{2}=\mathbb{E}\left\|\sum_{i=1}^{d} \gamma_{i} \sigma_{i}(\cdot, z(\cdot))\right\|_{L^{p}\left(\mathbb{T}^{N}\right)}^{2} \\
& \leq\left(\mathbb{E} \|\left.\sum_{i=1}^{\frac{2}{p}} \gamma_{i} \sigma_{i}(\cdot, z(\cdot))\right|_{L^{p}\left(\mathbb{T}^{N}\right)} ^{p}=\left(\int_{\mathbb{T}^{N}} \mathbb{E}\left|\sum_{i=1}^{d} \gamma_{i} \sigma_{i}(y, z(y))\right|^{p} \mathrm{~d} y\right)^{\frac{2}{p}}\right. \\
& \quad=C_{p}^{2}\left(\int_{\mathbb{T}^{N}}\left(\sum_{i=1}^{d} \mid \sigma_{i}\left(y,\left.z(y)\right|^{2}\right)^{\frac{2}{2}} \mathrm{~d} y\right)^{\frac{2}{p}} \leq C\left(\int_{\mathbb{T}^{N}}\left(1+|z(y)|^{2}\right)^{\frac{p}{2}} \mathrm{~d} y\right)^{\frac{2}{p}}\right. \\
& \quad \leq C\left(1+\|z\|_{L^{p}\left(\mathbb{T}^{N}\right)}^{2}\right)
\end{aligned}
$$


and the claim follows. In this paper, the letter $C$ denotes a positive constant, which is unimportant and may change from one line to another.

Let us close this section by stating the main result to be proved precisely.

Theorem 2.1. Let $p \in[2, \infty), q \in(2, \infty), m \in \mathbb{N}$. We suppose that

$$
u_{0} \in L^{q}\left(\Omega ; W^{m, p}\left(\mathbb{T}^{N}\right)\right) \cap L^{m q}\left(\Omega ; W^{1, m p}\left(\mathbb{T}^{N}\right)\right)
$$

and

$$
f_{\alpha} \in C^{m}(\mathbb{R}) \cap C^{2 l-1}(\mathbb{R}), \quad|\alpha| \leq 2 l-1 ; \quad \sigma_{i} \in C^{m}\left(\mathbb{T}^{N} \times \mathbb{R}\right), \quad i=1, \ldots, d,
$$

have bounded derivatives up to order $m$. Then there exists a unique solution to (1.1) which belongs to

$$
L^{q}\left(\Omega ; C\left([0, T] ; W^{m, p}\left(\mathbb{T}^{N}\right)\right)\right) \cap L^{m q}\left(\Omega ; C\left([0, T] ; W^{1, m p}\left(\mathbb{T}^{N}\right)\right)\right)
$$

and the following estimate holds true

$$
\begin{aligned}
& \mathbb{E} \sup _{0 \leq t \leq T}\|u(t)\|_{W^{m, p}\left(\mathbb{T}^{N}\right)}^{q}+\mathbb{E} \sup _{0 \leq t \leq T}\|u(t)\|_{W^{1, m p}\left(\mathbb{T}^{N}\right)}^{m q} \\
& \quad \leq C\left(1+\mathbb{E}\left\|u_{0}\right\|_{W^{m, p}\left(\mathbb{T}^{N}\right)}^{q}+\mathbb{E}\left\|u_{0}\right\|_{W^{1, m p}\left(\mathbb{T}^{N}\right)}^{m q}\right)
\end{aligned}
$$

Corollary 2.2. Let $k \in \mathbb{N}_{0}$ and $u_{0} \in L^{q}\left(\Omega ; C^{k+1}\left(\mathbb{T}^{N}\right)\right)$ for all $q \in(2, \infty)$. Assume that

$f_{\alpha} \in C^{k+1}(\mathbb{R}) \cap C^{2 l-1}(\mathbb{R}),|\alpha| \leq 2 l-1 ; \quad \sigma_{i} \in C^{k+1}\left(\mathbb{T}^{N} \times \mathbb{R}\right), i=1, \ldots, d$, have bounded derivatives up to order $k+1$. Then there exists a solution to (1.1) which belongs to

$$
L^{q}\left(\Omega ; C\left([0, T] ; C^{k, \lambda}\left(\mathbb{T}^{N}\right)\right)\right) \quad \text { for every } \quad \lambda \in(0,1) .
$$

Remark 2.3. In the proof, we show regularity of the mild solution, however, the resulting estimates imply that it is even strong (see [5] for a thorough exposition of these two concepts of solution).

\section{Preliminaries}

For the reader's convenience we shall first restate the following auxiliary result which is taken from [18, Theorem 5.2.5].

Proposition 3.1. Let $m \in \mathbb{N}, m \geq 2, p \in[1, \infty)$. Suppose that the function $G \in$ $C^{m}(\mathbb{R})$ has bounded derivatives up to order $m$. If $h \in W^{m, p}\left(\mathbb{T}^{N}\right) \cap W^{1, m p}\left(\mathbb{T}^{N}\right)$ then the following estimate holds true

$$
\|G(h)\|_{W^{m, p}\left(\mathbb{T}^{N}\right)} \leq C\left(1+\|h\|_{W^{1, m p}\left(\mathbb{T}^{N}\right)}^{m}+\|h\|_{W^{m, p}\left(\mathbb{T}^{N}\right)}\right)
$$

with a constant independent of $h$.

Proof. Since $G$ has a linear growth we have

$$
\|G(h)\|_{L^{p}\left(\mathbb{T}^{N}\right)} \leq C\left(1+\|h\|_{L^{p}\left(\mathbb{T}^{N}\right)}\right) .
$$


Next, we will employ the chain rule formula for partial derivatives of compositions:

$$
\mathrm{D}^{\beta} G(h(x))=\sum_{l=1}^{|\beta|} \sum_{\substack{\alpha_{1}+\cdots+\alpha_{l}=\beta \\\left|\alpha_{i}\right| \neq 0}} C_{\beta, l, \alpha_{1}, \ldots, \alpha_{l}} G^{(l)}(h(x)) \mathrm{D}^{\alpha_{1}} h(x) \cdots \mathrm{D}^{\alpha_{l}} h(x),
$$

where $\beta=\left(\beta_{1}, \ldots, \beta_{N}\right), \alpha_{i}=\left(\alpha_{i}^{1}, \ldots, \alpha_{i}^{N}\right), i=1, \ldots, l$, are multiindices and $C_{\beta, l, \alpha_{1}, \ldots, \alpha_{l}}$ are certain combinatorial constants. It is sufficient to consider $|\beta|=$ $m$. By the Hölder inequality we obtain

$$
\left\|G^{(l)}(h) \mathrm{D}^{\alpha_{1}} h \cdots \mathrm{D}^{\alpha_{l}} h\right\|_{L^{p}\left(\mathbb{T}^{N}\right)} \leq\left\|G^{(l)}\right\|_{L^{\infty}(\mathbb{R})} \prod_{i=1}^{l}\left\|\mathrm{D}^{\alpha_{i}} h\right\|_{L^{\frac{m p}{\left|\alpha_{i}\right|}\left(\mathbb{T}^{N}\right)}} .
$$

Due to interpolation inequalities, we have

$$
\|h\|_{W^{\left|\alpha_{i}\right|, \frac{m p}{\left|\alpha_{i}\right|}\left(\mathbb{T}^{N}\right)}} \leq C\|h\|_{W^{1, m p}\left(\mathbb{T}^{N}\right)}^{1-\theta_{i}}\|h\|_{W^{m, p}\left(\mathbb{T}^{N}\right)}^{\theta_{i}} \quad \text { with } \quad \theta_{i}=\frac{\left|\alpha_{i}\right|-1}{m-1} .
$$

Therefore

$$
\begin{aligned}
\left\|\mathrm{D}^{\beta} G(h)\right\|_{L^{p}\left(\mathbb{T}^{N}\right)} & \leq C \max _{1 \leq l \leq m} \sum_{\substack{\alpha_{1}+\cdots+\alpha_{l}=\beta \\
\left|\alpha_{i}\right| \neq 0}} \prod_{i=1}^{l}\|h\|_{W^{1, m p}\left(\mathbb{T}^{N}\right)}^{1-\theta_{i}}\|h\|_{W^{m, p}\left(\mathbb{T}^{N}\right)}^{\theta_{i}} \\
& \leq C \max _{1 \leq l \leq m}\|h\|_{W^{1, m p}\left(\mathbb{T}^{N}\right)}^{l-\frac{m-l}{m-1}}\|h\|_{W^{m, p}\left(\mathbb{T}^{N}\right)}^{\frac{m-l}{m-1}} \\
& \leq C\left(\|h\|_{W^{1, m p}\left(\mathbb{T}^{N}\right)}^{m}+\|h\|_{W^{m, p}\left(\mathbb{T}^{N}\right)}\right),
\end{aligned}
$$

where we used the fact that the function $y \mapsto a^{y}(b / a)^{\frac{m-y}{m-1}}$ is monotone so the maximal value is attained at $y=1$ or $y=m$. The proof is complete.

This result can be easily extended to more general outer function.

Corollary 3.2. Let $m \in \mathbb{N}, m \geq 2, p \in[1, \infty)$. Suppose that the function $G \in$ $C^{m}\left(\mathbb{T}^{N} \times \mathbb{R}\right)$ has bounded derivatives up to order $m$. If $h \in W^{m, p}\left(\mathbb{T}^{N}\right) \cap$ $W^{1, m p}\left(\mathbb{T}^{N}\right)$ then the following estimate holds true

$$
\|G(\cdot, h(\cdot))\|_{W^{m, p}\left(\mathbb{T}^{N}\right)} \leq C\left(1+\|h\|_{W^{1, m p}\left(\mathbb{T}^{N}\right)}^{m}+\|h\|_{W^{m, p}\left(\mathbb{T}^{N}\right)}\right)
$$

with a constant independent of $h$.

Remark 3.3. The situation is much easier for the first order derivatives: fix $p \in[1, \infty)$ and let $h \in W^{1, p}\left(\mathbb{T}^{N}\right)$

(i) if $G \in C^{1}(\mathbb{R})$ has a bounded derivative then

$$
\|G(h)\|_{W^{1, p}\left(\mathbb{T}^{N}\right)} \leq C\left(1+\|h\|_{W^{1, p}\left(\mathbb{T}^{N}\right)}\right),
$$

(ii) if $G \in C^{1}\left(\mathbb{T}^{N} \times \mathbb{R}\right)$ has bounded derivatives then

$$
\|G(\cdot, h(\cdot))\|_{W^{1, p}\left(\mathbb{T}^{N}\right)} \leq C\left(1+\|h\|_{W^{1, p}\left(\mathbb{T}^{N}\right)}\right),
$$

where the constant $C$ is independent of $h$. 


\section{Proof of the main result}

Let us review the main ideas of the proof. The proof is divided into three steps. In the first step, we apply the Banach fixed point theorem to conclude the existence of an $L^{m p}\left(\mathbb{T}^{N}\right)$-valued mild solution of (1.1). In the second step, we study Picard iterations for (1.1) and find a uniform estimate of the $W^{1, m p}\left(\mathbb{T}^{N}\right)$ norm. It is then used in the third step to derive a uniform estimate of the $W^{m, p}\left(\mathbb{T}^{N}\right)$-norm. This estimate remains valid also for the limit process and the statement follows.

These steps will be stated as propositions.

Proposition 4.1. (Fixed point argument) Let $p, q \in[2, \infty)$. Assume that $u_{0} \in$ $L^{q}\left(\Omega ; L^{p}\left(\mathbb{T}^{N}\right)\right)$ and

$$
f_{\alpha} \in C^{2 l-1}(\mathbb{R}), \quad|\alpha| \leq 2 l-1 ; \quad \sigma_{i} \in C^{1}\left(\mathbb{T}^{N} \times \mathbb{R}\right), \quad i=1, \ldots, d,
$$

have bounded derivatives of first order. Then there exists a unique mild solution to (1.1) which belongs to

$$
L^{q}\left(\Omega \times[0, T], \mathcal{P}, \mathrm{d} \mathbb{P} \otimes \mathrm{d} t ; L^{p}\left(\mathbb{T}^{N}\right)\right) .
$$

Proof. Let us denote

$$
\mathscr{H}=L^{q}\left(\Omega \times[0, T], \mathcal{P}, \mathrm{d} \mathbb{P} \otimes \mathrm{d} t ; L^{p}\left(\mathbb{T}^{N}\right)\right)
$$

and define the mapping

$$
\begin{aligned}
(\mathscr{K} v)(t) & =\mathcal{S}_{p}(t) u_{0}+\int_{0}^{t} \mathcal{S}_{p}(t-s) F(v(s)) \mathrm{d} s+\int_{0}^{t} \mathcal{S}_{p}(t-s) \sigma(v(s)) \mathrm{d} W(s) \\
& =\mathcal{S}_{p}(t) u_{0}+\left(\mathscr{K}_{1} v\right)(t)+\left(\mathscr{K}_{2} v\right)(t), \quad t \in[0, T], v \in \mathscr{H} .
\end{aligned}
$$

Here, we employ stochastic integration in $L^{p}\left(\mathbb{T}^{N}\right)$ as introduced in Sect. 2. We shall prove that $\mathscr{K}$ maps $\mathscr{H}$ into $\mathscr{H}$ and that it is a contraction.

Since $u_{0} \in L^{q}\left(\Omega ; L^{p}\left(\mathbb{T}^{N}\right)\right)$ it follows easily that $\mathcal{S}_{p}(t) u_{0} \in \mathscr{H}$. In order to estimate the second term, let $\delta=\frac{2 l-1}{2 l}$ and note that

$$
\mathcal{S}_{p}(t-s) F(v(s))=\mathcal{S}_{p}(t-s)\left(-\mathcal{A}_{p}\right)^{\delta}\left(-\mathcal{A}_{p}\right)^{-\delta} \sum_{\substack{|\alpha| \leq 2 l-1 \\ a_{\alpha} \neq 0}} a_{\alpha} \mathrm{D}^{\alpha} f_{\alpha}(v(s))
$$

where the operator $\left(-\mathcal{A}_{p}\right)^{\delta}$ commutes with the semigroup and the operator

$$
\begin{aligned}
& \mathcal{B}_{p}: L^{p}\left(\mathbb{T}^{N} ; \mathbb{R}^{\eta}\right) \longrightarrow L^{p}\left(\mathbb{T}^{N}\right) \\
& \left\{z_{\alpha}\right\}_{|\alpha| \leq 2 l-1 a_{\alpha} \neq 0} \longmapsto\left(-\mathcal{A}_{p}\right)^{-\delta} \sum_{\substack{|\alpha| \leq 2 l-1 \\
a_{\alpha} \neq 0}} a_{\alpha} \mathrm{D}^{\alpha} z_{\alpha}
\end{aligned}
$$

is bounded. Indeed, let $p^{*}$ be the conjugate exponent to $p$. Then the operator $L^{p^{*}}\left(\mathbb{T}^{N}\right) \rightarrow L^{p^{*}}\left(\mathbb{T}^{N}\right), v \mapsto a_{\alpha} \mathrm{D}^{\alpha}\left(-\mathcal{A}_{p^{*}}\right)^{-\delta} v,|\alpha| \leq 2 l-1$, is clearly bounded 
so for $z \in L^{p}\left(\mathbb{T}^{N} ; \mathbb{R}^{\eta}\right)$ we have

$$
\begin{aligned}
& \left\|\left(-\mathcal{A}_{p}\right)^{-\delta} \sum_{\substack{|\alpha| \leq 2 l-1 \\
a_{\alpha} \neq 0}} a_{\alpha} \mathrm{D}^{\alpha} z_{\alpha}\right\|_{\substack{L^{p}\left(\mathbb{T}^{N}\right)\\
}} \\
& =\sup _{\substack{v \in L^{p^{*}}\left(\mathbb{T}^{N}\right) \\
\|v\|_{L^{p^{*}}\left(\mathbb{T}^{N}\right)} \leq 1}}\left|\int_{\mathbb{T}^{N}}\left(-\mathcal{A}_{p}\right)^{-\delta} \sum_{\substack{|\alpha| \leq 2 l-1 \\
a_{\alpha} \neq 0}} a_{\alpha} \mathrm{D}^{\alpha} z_{\alpha}(x) v(x) \mathrm{d} x\right| \\
& =\sup _{\substack{v \in L^{p^{*}}\left(\mathbb{T}^{N}\right) \\
\|v\|_{L^{p^{*}}\left(\mathbb{T}^{N}\right)} \leq 1}}\left|\sum_{\substack{|\alpha| \leq 2 l-1 \\
a_{\alpha} \neq 0}} \int_{\mathbb{T}^{N}} z_{\alpha}(x) a_{\alpha} \mathrm{D}^{\alpha}\left(-\mathcal{A}_{p^{*}}\right)^{-\delta} v(x) \mathrm{d} x\right| \\
& =\sup _{\substack{v \in L^{p^{*}}\left(\mathbb{T}^{N}\right) \\
\|v\|_{L^{p^{*}}\left(\mathbb{T}^{N}\right)} \leq 1}}\left|\int_{\mathbb{T}^{N}}\left\langle z(x),\left\{a_{\alpha} \mathrm{D}^{\alpha}\left(-\mathcal{A}_{p^{*}}\right)^{-\delta} v(x)\right\} \begin{array}{c}
|\alpha| \leq 2 l-1 \\
a_{\alpha} \neq 0
\end{array}\right\rangle_{\mathbb{R}^{\eta}} \mathrm{d} x\right|
\end{aligned}
$$

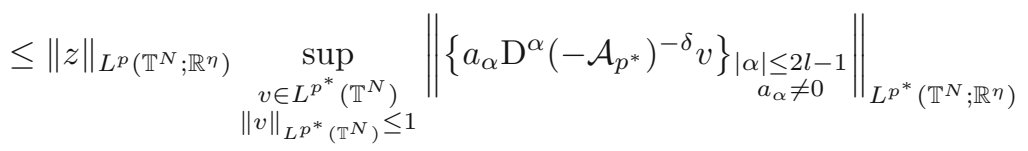

$$
\begin{aligned}
& \leq C\|z\|_{L^{p}\left(\mathbb{T}^{N} ; \mathbb{R}^{\eta}\right)}
\end{aligned}
$$

and the claim follows. Next, all $f_{\alpha},|\alpha| \leq 2 l-1$, have bounded derivatives hence at most linear growth, so it holds for any $z \in L^{p}\left(\mathbb{T}^{N}\right)$

$$
\|f(z)\|_{L^{p}\left(\mathbb{T}^{N} ; \mathbb{R}^{\eta}\right)} \leq C\left(1+\|z\|_{L^{p}\left(\mathbb{T}^{N}\right)}\right) .
$$

Later on, if there is no danger of confusion we will write $L^{p}\left(\mathbb{T}^{N}\right)$ instead of $L^{p}\left(\mathbb{T}^{N} ; \mathbb{R}^{\eta}\right)$. Let $v \in \mathscr{H}$, then using the above remark, the fact $(2.1)$, the estimate (4.1) and the Young inequality for convolutions we obtain

$$
\begin{aligned}
\left\|\mathscr{K}_{1} v\right\|_{\mathscr{H}}^{q} & =\mathbb{E} \int_{0}^{T}\left\|\int_{0}^{t} \mathcal{S}_{p}(t-s) F(v(s)) \mathrm{d} s\right\|_{L^{p}\left(\mathbb{T}^{N}\right)}^{q} \mathrm{~d} t \\
& \leq \mathbb{E} \int_{0}^{T}\left(\int_{0}^{t}\left\|\left(-\mathcal{A}_{p}\right)^{\delta} \mathcal{S}_{p}(t-s) \mathcal{B}_{p} f(v(s))\right\|_{L^{p}\left(\mathbb{T}^{N}\right)} \mathrm{d} s\right)^{q} \mathrm{~d} t \\
& \leq C \mathbb{E} \int_{0}^{T}\left(\int_{0}^{t} \frac{1}{(t-s)^{\delta}}\left\|\mathcal{B}_{p} f(v(s))\right\|_{L^{p}\left(\mathbb{T}^{N}\right)} \mathrm{d} s\right)^{q} \mathrm{~d} t \\
& \leq C \mathbb{E} \int_{0}^{T}\left(\int_{0}^{t} \frac{1}{(t-s)^{\delta}}\|f(v(s))\|_{L^{p}\left(\mathbb{T}^{N}\right)} \mathrm{d} s\right)^{q} \mathrm{~d} t
\end{aligned}
$$




$$
\begin{aligned}
& \leq C \mathbb{E} \int_{0}^{T}\left(\int_{0}^{t} \frac{1}{(t-s)^{\delta}}\left(1+\|v(s)\|_{L^{p}\left(\mathbb{T}^{N}\right)}\right) \mathrm{d} s\right)^{q} \mathrm{~d} t \\
& \leq C T^{q(1-\delta)} \mathbb{E} \int_{0}^{T}\left(1+\|v(s)\|_{L^{p}\left(\mathbb{T}^{N}\right)}\right)^{q} \mathrm{~d} s=C T^{q(1-\delta)}\left(T+\|v\|_{\mathscr{H}}^{q}\right) .
\end{aligned}
$$

Next, by the Burkholder-Davis-Gundy inequality for martingales with values in 2-smooth Banach spaces (see $[3,16]$ ), we have

$$
\begin{aligned}
\left\|\mathscr{K}_{2} v\right\|_{\mathscr{H}}^{q} & =\mathbb{E} \int_{0}^{T}\left\|\int_{0}^{t} \mathcal{S}_{p}(t-s) \sigma(v(s)) \mathrm{d} W(s)\right\|_{L^{p}\left(\mathbb{T}^{N}\right)}^{q} \mathrm{~d} t \\
& \leq C \int_{0}^{T} \mathbb{E}\left(\int_{0}^{t}\left\|\mathcal{S}_{p}(t-s) \sigma(v(s))\right\|_{\gamma\left(\mathfrak{U} ; L^{p}\left(\mathbb{T}^{N}\right)\right)}^{2} \mathrm{~d} s\right)^{\frac{q}{2}} \mathrm{~d} t \\
& \leq C T^{\frac{q-2}{2}} \int_{0}^{T} \mathbb{E} \int_{0}^{t}\|\sigma(v(s))\|_{\gamma\left(\mathfrak{U} ; L^{p}\left(\mathbb{T}^{N}\right)\right)}^{q} \mathrm{~d} s \mathrm{~d} t .
\end{aligned}
$$

The $\gamma$-radonifying norm can be computed, for almost every $s$ and $\omega$, using (2.3) as in (2.4). Therefore

$$
\left\|\mathscr{K}_{2} v\right\|_{\mathscr{H}}^{q} \leq C T^{\frac{q-2}{2}} \int_{0}^{T} \mathbb{E} \int_{0}^{t}\left(1+\|v(s)\|_{L^{p}\left(\mathbb{T}^{N}\right)}^{q}\right) \mathrm{d} s \mathrm{~d} t \leq C T^{\frac{q}{2}}\left(T+\|v\|_{\mathscr{H}}^{q}\right) .
$$

We conclude that $\mathscr{K}(\mathscr{H}) \subset \mathscr{H}$ for any $T>0$.

In order to show the contraction property of $\mathscr{K}_{1}$, we will mimic the procedure from (4.2) and use the Lipschitz continuity of $f$. Indeed, $f_{\alpha},|\alpha| \leq 2 l-1$, have bounded derivatives so they are Lipschitz continuous and

$$
\left\|f\left(z_{1}\right)-f\left(z_{2}\right)\right\|_{L^{p}\left(\mathbb{T}^{N}\right)} \leq C\left\|z_{1}-z_{2}\right\|_{L^{p}\left(\mathbb{T}^{N}\right)}, \quad z_{1}, z_{2} \in L^{p}\left(\mathbb{T}^{N}\right),
$$

can be proved as (4.1). For $v, w \in \mathscr{H}$

$$
\begin{aligned}
& \left\|\mathscr{K}_{1} v-\mathscr{K}_{1} w\right\|_{\mathscr{H}}^{q} \\
& =\mathbb{E} \int_{0}^{T}\left\|\int_{0}^{t} \mathcal{S}_{p}(t-s)(F(v(s))-F(w(s))) \mathrm{d} s\right\|_{L^{p}\left(\mathbb{T}^{N}\right)}^{q} \mathrm{~d} t \\
& \leq \mathbb{E} \int_{0}^{T}\left(\int_{0}^{t}\left\|\left(-\mathcal{A}_{p}\right)^{\delta} \mathcal{S}_{p}(t-s) \mathcal{B}_{p}(f(v(s))-f(w(s)))\right\|_{L^{p}\left(\mathbb{T}^{N}\right)} \mathrm{d} s\right)^{q} \mathrm{~d} t \\
& \leq C \mathbb{E} \int_{0}^{T}\left(\int_{0}^{t} \frac{1}{(t-s)^{\delta}} \| \mathcal{B}_{p}\left(f(v(s))-f(w(s)) \|_{L^{p}\left(\mathbb{T}^{N}\right)} \mathrm{d} s\right)^{q} \mathrm{~d} t\right.
\end{aligned}
$$




$$
\begin{aligned}
& \leq C \mathbb{E} \int_{0}^{T}\left(\int_{0}^{t} \frac{1}{(t-s)^{\delta}}\|f(v(s))-f(w(s))\|_{L^{p}\left(\mathbb{T}^{N}\right)} \mathrm{d} s\right)^{q} \mathrm{~d} t \\
& \leq C \mathbb{E} \int_{0}^{T}\left(\int_{0}^{t} \frac{1}{(t-s)^{\delta}}\|v(s)-w(s)\|_{L^{p}\left(\mathbb{T}^{N}\right)} \mathrm{d} s\right)^{q} \mathrm{~d} t \\
& \leq C T^{q(1-\delta)} \mathbb{E} \int_{0}^{T}\|v(s)-w(s)\|_{L^{p}\left(\mathbb{T}^{N}\right)}^{q} \mathrm{~d} s=C T^{q(1-\delta)}\|v-w\|_{\mathscr{H}}^{q} .
\end{aligned}
$$

In the case of $\mathscr{K}_{2}$ we employ the same calculations as in (4.3) and the sequel:

$$
\begin{aligned}
& \left\|\mathscr{K}_{2} v-\mathscr{K}_{2} w\right\|_{\mathscr{H}}^{q} \\
& =\mathbb{E} \int_{0}^{T}\left\|\int_{0}^{t} \mathcal{S}_{p}(t-s)(\sigma(v(s))-\sigma(w(s))) \mathrm{d} W(s)\right\|_{L^{p}\left(\mathbb{T}^{N}\right)}^{q} \mathrm{~d} t \\
& \leq C \int_{0}^{T} \mathbb{E}\left(\int_{0}^{t}\left\|\mathcal{S}_{p}(t-s)(\sigma(v(s))-\sigma(w(s)))\right\|_{\gamma\left(\mathfrak{U} ; L^{p}\left(\mathbb{T}^{N}\right)\right)}^{2} \mathrm{~d} s\right)^{\frac{q}{2}} \mathrm{~d} t \\
& \leq C T^{\frac{q-2}{2}} \int_{0}^{T} \mathbb{E} \int_{0}^{t}\|\sigma(v(s))-\sigma(w(s))\|_{\gamma\left(\mathfrak{U} ; L^{p}\left(\mathbb{T}^{N}\right)\right)}^{q} \mathrm{~d} s \mathrm{~d} t .
\end{aligned}
$$

Let $z_{1}, z_{2} \in L^{p}\left(\mathbb{T}^{N}\right)$. Then for the $\gamma$-radonifying norm we have

$$
\begin{aligned}
\left\|\sigma\left(z_{1}\right)-\sigma\left(z_{2}\right)\right\|_{\gamma\left(\mathfrak{U} ; L^{p}\left(\mathbb{T}^{N}\right)\right)}^{q} & \\
\leq & \left(\mathbb{E}\left\|\sum_{i=1}^{d} \gamma_{i}\left(\sigma_{i}\left(\cdot, z_{1}(\cdot)\right)-\sigma_{i}\left(\cdot, z_{2}(\cdot)\right)\right)\right\|_{L^{p}\left(\mathbb{T}^{N}\right)}^{2}\right)^{\frac{q}{2}} \\
& \leq\left(\mathbb{E}\left\|\sum_{i=1}^{d} \gamma_{i}\left(\sigma_{i}\left(\cdot, z_{1}(\cdot)\right)-\sigma_{i}\left(\cdot, z_{2}(\cdot)\right)\right)\right\|_{L^{p}\left(\mathbb{T}^{N}\right)}^{p}\right)^{\frac{q}{p}} \\
& =C\left(\int_{\mathbb{T}^{N}}\left(\sum_{i=1}^{d}\left|\sigma_{i}\left(y, z_{1}(y)\right)-\sigma_{i}\left(y, z_{2}(y)\right)\right|^{2}\right)^{\frac{p}{2}} \mathrm{~d} y\right)^{\frac{q}{p}} \\
& \leq C\left\|z_{1}-z_{2}\right\|_{L^{p}\left(\mathbb{T}^{N}\right)}^{q},
\end{aligned}
$$

where the last inequality follows from the fact that all $\sigma_{i}, i=1, \ldots, d$, have bounded derivatives and therefore are Lipschitz continuous. We conclude

$$
\left\|\mathscr{K}_{2} v-\mathscr{K}_{2} w\right\|_{\mathscr{H}}^{q} \leq C T^{\frac{q}{2}}\|v-w\|_{\mathscr{H}}^{q} .
$$

Consequently

$$
\|\mathscr{K} v-\mathscr{K} w\|_{\mathscr{H}} C\left(T^{1-\delta}+T^{\frac{1}{2}}\right)\|v-w\|_{\mathscr{H}},
$$


where the constant does not depend on $T$ and $u_{0}$. Therefore, if

$$
C\left(T^{1-\delta}+T^{\frac{1}{2}}\right)<1
$$

then the mapping $\mathscr{K}$ has unique fixed point $u$ in $\mathscr{H}$ which is a mild solution of (1.1). Furthermore, by a standard use of the factorization lemma, it has continuous trajectories with values in $L^{p}\left(\mathbb{T}^{N}\right)$, i.e. belongs to

$$
L^{q}\left(\Omega ; C\left([0, T] ; L^{p}\left(\mathbb{T}^{N}\right)\right)\right) .
$$

Therefore, the condition on $T$ can be easily removed by considering the equation on intervals $[0, \tilde{T}],[\tilde{T}, 2 \tilde{T}], \ldots$ with $\tilde{T}$ satisfying (4.4).

The estimates from previous proposition can be improved in order to obtain a better regularity of $u$.

Proposition 4.2. (Estimate in $\left.W^{1, p}\left(\mathbb{T}^{N}\right)\right)$ Let $p \in[2, \infty), q \in(2, \infty)$. Assume that $u_{0} \in L^{q}\left(\Omega ; W^{1, p}\left(\mathbb{T}^{N}\right)\right)$ and

$$
f_{\alpha} \in C^{2 l-1}(\mathbb{R}), \quad|\alpha| \leq 2 l-1 ; \quad \sigma_{i} \in C^{1}\left(\mathbb{T}^{N} \times \mathbb{R}\right), \quad i=1, \ldots, d,
$$

have bounded derivatives of first order. Then the mild solution of (1.1) belongs to

$$
L^{q}\left(\Omega ; C\left([0, T] ; W^{1, p}\left(\mathbb{T}^{N}\right)\right)\right)
$$

and the following estimate holds true

$$
\mathbb{E} \sup _{0 \leq t \leq T}\|u(t)\|_{W^{1, p}\left(\mathbb{T}^{N}\right)}^{q} \leq C\left(1+\mathbb{E}\left\|u_{0}\right\|_{W^{1, p}\left(\mathbb{T}^{N}\right)}^{q}\right) .
$$

Proof. Recall that $u$ is the limit of Picard iterations: let $u^{0}(t)=u_{0}$ and for $n \in \mathbb{N}$ define

$$
\begin{aligned}
u^{n}(t)= & \mathcal{S}_{p}(t) u_{0}+\int_{0}^{t} \mathcal{S}_{p}(t-s) F\left(u^{n-1}(s)\right) \mathrm{d} s \\
& +\int_{0}^{t} \mathcal{S}_{p}(t-s) \sigma\left(u^{n-1}(s)\right) \mathrm{d} W(s) .
\end{aligned}
$$

We will show

$$
\mathbb{E} \sup _{0 \leq t \leq T}\left\|u^{n}(t)\right\|_{W^{1, p}\left(\mathbb{T}^{N}\right)}^{q} \leq C\left(1+\mathbb{E}\left\|u_{0}\right\|_{W^{1, p}\left(\mathbb{T}^{N}\right)}^{q}\right), \quad \forall n \in \mathbb{N},
$$

with a constant $C$ independent of $n$. By induction on $n$, assume that the hypothesis is satisfied for $u^{n-1}$ and compute the estimate for $u^{n}$. We will proceed term by term and follow the ideas of Proposition 4.1. Consider the operators $\mathcal{S}_{p}(t), t \geq 0$, restricted to the Sobolev space $W^{1, p}\left(\mathbb{T}^{N}\right)$ and denote them by $\mathcal{S}_{1, p}(t), t \geq 0$. These operators form a bounded analytic semigroup on 
$W^{1, p}\left(\mathbb{T}^{N}\right)$ generated by the part of $\mathcal{A}_{p}$ in $W^{1, p}\left(\mathbb{T}^{N}\right)$ (see [2, Theorem V.2.1.3]). Let us denote this generator by $\mathcal{A}_{1, p}$. Therefore we have

$$
\begin{aligned}
\mathbb{E} \sup _{0 \leq t \leq T}\left\|\mathcal{S}_{p}(t) u_{0}\right\|_{W^{1, p}\left(\mathbb{T}^{N}\right)}^{q} & =\mathbb{E} \sup _{0 \leq t \leq T}\left\|\mathcal{S}_{1, p}(t) u_{0}\right\|_{W^{1, p}\left(\mathbb{T}^{N}\right)}^{q} \\
& \leq C \mathbb{E}\left\|u_{0}\right\|_{W^{1, p}\left(\mathbb{T}^{N}\right)}^{q} .
\end{aligned}
$$

As above, let $\delta=\frac{2 l-1}{2 l}$ and consider the operator

$$
\begin{aligned}
& \mathcal{B}_{1, p}: W^{1, p}\left(\mathbb{T}^{N} ; \mathbb{R}^{\eta}\right) \longrightarrow W^{1, p}\left(\mathbb{T}^{N}\right) \\
&\left\{z_{\alpha}\right\}_{\substack{|\alpha| \leq 2 l-1 \\
a_{\alpha} \neq 0}} \longmapsto\left(-\mathcal{A}_{p}\right)^{-\delta} \sum_{\substack{|\alpha| \leq 2 l-1 \\
a_{\alpha} \neq 0}} a_{\alpha} \mathrm{D}^{\alpha} z_{\alpha} .
\end{aligned}
$$

We will show that it is a bounded operator. Indeed, according to the computations in the proof of Proposition 4.1, for any $z \in W^{1, p}\left(\mathbb{T}^{N} ; \mathbb{R}^{\eta}\right)$,

$$
\left\|\mathcal{B}_{1, p} z\right\|_{L^{p}\left(\mathbb{T}^{N}\right)} \leq C\|z\|_{L^{p}\left(\mathbb{T}^{N} ; \mathbb{R}^{\eta}\right)} .
$$

For any multiindex $\beta=\left(\beta_{1}, \ldots, \beta_{N}\right)$ such that $|\beta|=1$, we can write

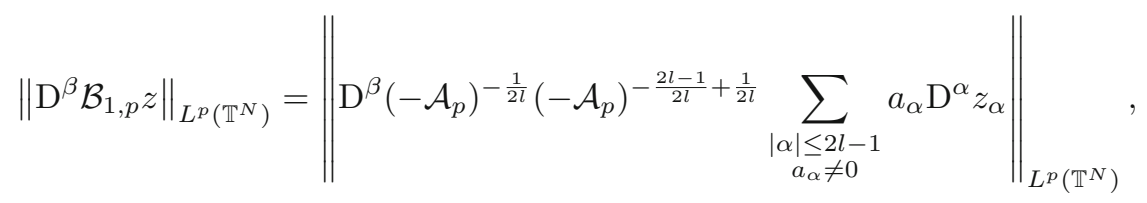

where the operator $L^{p}\left(\mathbb{T}^{N}\right) \rightarrow L^{p}\left(\mathbb{T}^{N}\right), v \mapsto \mathrm{D}^{\beta}\left(-\mathcal{A}_{p}\right)^{-\frac{1}{2 l}} v$, is bounded. For each $\alpha,|\alpha| \leq 2 l-1$, let us fix a multiindex $\alpha^{\prime}$ such that it is of order 1 and $\alpha-\alpha^{\prime}$ is also a multiindex, i.e. $\left|\alpha^{\prime}\right|=1$ and $\left|\alpha-\alpha^{\prime}\right|=|\alpha|-1$. Note, that if $p^{*}$ is the conjugate exponent to $p$, the operator $L^{p^{*}}\left(\mathbb{T}^{N}\right) \rightarrow L^{p^{*}}\left(\mathbb{T}^{N}\right), v \mapsto$ $a_{\alpha} \mathrm{D}^{\alpha-\alpha^{\prime}}\left(-\mathcal{A}_{p^{*}}\right)^{\frac{-2 l+2}{2 l}} v,|\alpha| \leq 2 l-1$, is bounded as well. We conclude

$$
\begin{aligned}
& \left\|\left(-\mathcal{A}_{p}\right)^{\frac{-2 l+2}{2 l}} \sum_{\substack{|\alpha| \leq 2 l-1 \\
a_{\alpha} \neq 0}} a_{\alpha} \mathrm{D}^{\alpha} z_{\alpha}\right\|_{L^{p}\left(\mathbb{T}^{N}\right)} \\
& =\sup _{\substack{v \in L^{p^{*}}\left(\mathbb{T}^{N}\right) \\
\|v\|_{L^{p^{*}}\left(\mathbb{T}^{N}\right)} \leq 1}}\left|\int_{\mathbb{T}^{N}}\left(-\mathcal{A}_{p}\right)^{\frac{-2 l+2}{2 l}} \sum_{\substack{|\alpha| \leq 2 l-1 \\
a_{\alpha} \neq 0}} a_{\alpha} \mathrm{D}^{\alpha} z_{\alpha}(x) v(x) \mathrm{d} x\right| \\
& =\sup _{\substack{v \in L^{p^{*}}\left(\mathbb{T}^{N}\right) \\
\|v\|_{L^{p^{*}}\left(\mathbb{T}^{N}\right)} \leq 1}}\left|\sum_{\substack{|\alpha| \leq 2 l-1 \\
a_{\alpha} \neq 0}} \int_{\mathbb{T}^{N}} \mathrm{D}^{\alpha^{\prime}} z_{\alpha}(x) a_{\alpha} \mathrm{D}^{\alpha-\alpha^{\prime}}\left(-\mathcal{A}_{p^{*}}\right)^{\frac{-2 l+2}{2 l}} v(x) \mathrm{d} x\right| \\
& \leq\left\|\left\{\mathrm{D}^{\alpha^{\prime}} z_{\alpha}\right\}_{\substack{|\alpha| \leq 2 l-1 \\
a_{\alpha} \neq 0}}\right\|_{L^{p}\left(\mathbb{T}^{N} ; \mathbb{R}^{\eta}\right)}
\end{aligned}
$$




$$
\begin{aligned}
& \times \sup _{\substack{v \in L^{p^{*}}\left(\mathbb{T}^{N}\right) \\
\|v\|_{L^{p^{*}}\left(\mathbb{T}^{N}\right)} \leq 1}}\left\|\left\{a_{\alpha} \mathrm{D}^{\alpha-\alpha^{\prime}}\left(-\mathcal{A}_{p^{*}}\right)^{\frac{-2 l+2}{2 l}} v\right\}_{\substack{|\alpha| \leq 2 l-1 \\
a_{\alpha} \neq 0}}\right\|_{L^{p^{*}\left(\mathbb{T}^{N} ; \mathbb{R}^{\eta}\right)}} \\
& \leq C\|z\|_{W^{1, p}\left(\mathbb{T}^{N} ; \mathbb{R}^{\eta}\right)}
\end{aligned}
$$

and the claim follows. Therefore, we have

$$
\begin{aligned}
& \mathbb{E} \sup _{0 \leq t \leq T}\left\|\int_{0}^{t} \mathcal{S}_{p}(t-s) F\left(u^{n-1}(s)\right) \mathrm{d} s\right\|_{W^{1, p}\left(\mathbb{T}^{N}\right)}^{q} \\
& \leq \mathbb{E} \sup _{0 \leq t \leq T}\left(\int_{0}^{t}\left\|\left(-\mathcal{A}_{p}\right)^{\delta} \mathcal{S}_{p}(t-s) \mathcal{B}_{1, p} f\left(u^{n-1}(s)\right)\right\|_{W^{1, p}\left(\mathbb{T}^{N}\right)} \mathrm{d} s\right)^{q} \\
& \leq \mathbb{E} \sup _{0 \leq t \leq T}\left(\int_{0}^{t}\left\|\left(-\mathcal{A}_{1, p}\right)^{\delta} \mathcal{S}_{1, p}(t-s) \mathcal{B}_{1, p} f\left(u^{n-1}(s)\right)\right\|_{W^{1, p}\left(\mathbb{T}^{N}\right)} \mathrm{d} s\right)^{q} \\
& \leq C \mathbb{E} \sup _{0 \leq t \leq T}\left(\int_{0}^{t} \frac{1}{(t-s)^{\delta}}\left\|f\left(u^{n-1}(s)\right)\right\|_{W^{1, p}\left(\mathbb{T}^{N}\right)} \mathrm{d} s\right)^{q} \\
& \leq C T^{q(1-\delta)} \sup _{0 \leq t \leq T}\left\|f\left(u^{n-1}(t)\right)\right\|_{W^{1, p}\left(\mathbb{T}^{N}\right)}^{q} \cdot
\end{aligned}
$$

To deduce a similar estimate for the stochastic term, we need to consider stochastic integration in $W^{1, p}\left(\mathbb{T}^{N}\right)$. Employing the Hölder inequality and the equivalence of norms on $W^{1, p}\left(\mathbb{T}^{N}\right)$ we obtain for $z \in W^{1, p}\left(\mathbb{T}^{N}\right)$

$$
\begin{aligned}
\|\sigma(z)\|_{\gamma\left(\mathfrak{U} ; W^{1, p}\left(\mathbb{T}^{N}\right)\right)}^{q} & =\left(\mathbb{E}\left\|\sum_{i=1}^{d} \gamma_{i} \sigma_{i}(\cdot, z(\cdot))\right\|_{W^{1, p}\left(\mathbb{T}^{N}\right)}^{2}\right)^{\frac{q}{2}} \\
& \leq\left(\mathbb{E}\left\|\sum_{i=1}^{d} \gamma_{i} \sigma_{i}(\cdot, z(\cdot))\right\|_{W^{1, p}\left(\mathbb{T}^{N}\right)}^{p}\right)^{\frac{q}{p}} \\
& \leq C\left(\mathbb{E}\left\|\sum_{i=1}^{d} \gamma_{i}\left(-\mathcal{A}_{p}\right)^{\frac{1}{2 l}} \sigma_{i}(\cdot, z(\cdot))\right\|_{L^{p}\left(\mathbb{T}^{N}\right)}^{p}\right)^{\frac{q}{p}} \\
& =C\left(\int_{\mathbb{T}^{N}}\left(\sum_{i=1}^{d}\left|\left(-\mathcal{A}_{p}\right)^{\frac{1}{2 l}} \sigma_{i}(y, z(y))\right|^{\frac{p}{2}} \mathrm{~d} y\right)^{\frac{p}{p}}\right. \\
& \leq C \sum_{i=1}^{d}\left\|\sigma_{i}(\cdot, z(\cdot))\right\|_{W^{1, p}\left(\mathbb{T}^{N}\right)}^{q} \cdot
\end{aligned}
$$

Since $q \in(2, \infty)$, we make use of the maximal estimate for stochastic convolution [3, Corollary 3.5] which can be proved by the factorization method. For the 
reader's convenience we recall the basic steps of the proof. Let $\vartheta \in(1 / q, 1 / 2)$, then according to the stochastic Fubini theorem [4, Proposition 3.3(v)],

$$
\int_{0}^{t} \mathcal{S}_{p}(t-s) \sigma\left(u^{n-1}(s)\right) \mathrm{d} W(s)=\frac{1}{\Gamma(\vartheta)} \int_{0}^{t}(t-s)^{\vartheta-1} \mathcal{S}_{p}(t-s) y(s) \mathrm{d} s,
$$

where

$$
y(s)=\frac{1}{\Gamma(1-\vartheta)} \int_{0}^{s}(s-r)^{-\vartheta} \mathcal{S}_{p}(s-r) \sigma\left(u^{n-1}(r)\right) \mathrm{d} W(r) .
$$

Hence application of the Hölder, Burkholder-Davis-Gundy and Young inequalities yields (here the constant $C$ is independent on $T$ )

$$
\begin{aligned}
& \mathbb{E} \sup _{0 \leq t \leq T}\left\|\int_{0}^{t} \mathcal{S}_{p}(t-s) \sigma\left(u^{n-1}(s)\right) \mathrm{d} W(s)\right\|_{W^{1, p}\left(\mathbb{T}^{N}\right)}^{q} \\
& \leq C T^{\frac{q}{2}-1} \mathbb{E} \int_{0}^{T}\left\|\sigma\left(u^{n-1}(s)\right)\right\|_{\gamma\left(\mathfrak{U} ; W^{1, p}\left(\mathbb{T}^{N}\right)\right)}^{q} \mathrm{~d} s
\end{aligned}
$$

SO

$$
\begin{aligned}
& \mathbb{E} \sup _{0 \leq t \leq T}\left\|\int_{0}^{t} \mathcal{S}_{p}(t-s) \sigma\left(u^{n-1}(s)\right) \mathrm{d} W(s)\right\|_{W^{1, p}\left(\mathbb{T}^{N}\right)}^{q} \\
& \leq C T^{\frac{q}{2}-1} \sum_{i=1}^{d} \mathbb{E} \int_{0}^{T}\left\|\sigma_{i}\left(\cdot, u^{n-1}(s, \cdot)\right)\right\|_{W^{1, p}\left(\mathbb{T}^{N}\right)}^{q} \mathrm{~d} s \\
& \leq C T^{\frac{q}{2}} \sum_{i=1}^{d} \mathbb{E} \sup _{0 \leq t \leq T}\left\|\sigma_{i}\left(\cdot, u^{n-1}(t, \cdot)\right)\right\|_{W^{1, p}\left(\mathbb{T}^{N}\right)}^{q}
\end{aligned}
$$

and finally

$$
\begin{aligned}
& \mathbb{E} \sup _{0 \leq t \leq T}\left\|u^{n}(t)\right\|_{W^{1, p}\left(\mathbb{T}^{N}\right)}^{q} \leq C \mathbb{E}\left\|u_{0}\right\|_{W^{1, p}\left(\mathbb{T}^{N}\right)}^{q} \\
& \quad+C T^{q(1-\delta)} \mathbb{E} \sup _{0 \leq t \leq T}\left\|f\left(u^{n-1}(t)\right)\right\|_{W^{1, p}\left(\mathbb{T}^{N}\right)}^{q} \\
& \quad+C T^{\frac{q}{2}} \sum_{i=1}^{d} \mathbb{E} \sup _{0 \leq t \leq T}\left\|\sigma_{i}\left(\cdot, u^{n-1}(t, \cdot)\right)\right\|_{W^{1, p}\left(\mathbb{T}^{N}\right)}^{q},
\end{aligned}
$$

where the constant does not depend on $n$. Now, we make use of Remark 3.3 and obtain

$$
\begin{aligned}
& \mathbb{E} \sup _{0 \leq t \leq T}\left\|u^{n}(t)\right\|_{W^{1, p}\left(\mathbb{T}^{N}\right)}^{q} \leq C \mathbb{E}\left\|u_{0}\right\|_{W^{1, p}\left(\mathbb{T}^{N}\right)}^{q} \\
& \quad+C\left(T^{q(1-\delta)}+T^{\frac{q}{2}}\right)\left(1+\mathbb{E} \sup _{0 \leq t \leq T}\left\|u^{n-1}(t)\right\|_{W^{1, p}\left(\mathbb{T}^{N}\right)}^{q}\right) .
\end{aligned}
$$


Let us make an additional hypothesis: assume that $T$ is such that

$$
C_{T}=C\left(T^{q(1-\delta)}+T^{\frac{q}{2}}\right)<1 .
$$

Denoting $K_{n}=\mathbb{E} \sup _{0 \leq t \leq T}\left\|u^{n}(t)\right\|_{W^{1, p}\left(\mathbb{T}^{N}\right)}^{q}, n \in \mathbb{N}_{0}$, we have

$$
K_{n} \leq C \mathbb{E}\left\|u_{0}\right\|_{W^{1, p}\left(\mathbb{T}^{N}\right)}^{q}+C_{T}\left(1+K_{n-1}\right)
$$

and inductively in $n$

$$
\mathbb{E} \sup _{0 \leq t \leq T}\left\|u^{n}(t)\right\|_{W^{1, p}\left(\mathbb{T}^{N}\right)}^{q} \leq \tilde{C}_{T}\left(1+\mathbb{E}\left\|u_{0}\right\|_{W^{1, p}\left(\mathbb{T}^{N}\right)}^{q}\right),
$$

where $\tilde{C}_{T}$ is independent $n$. So (4.6) follows if $T$ is sufficiently small.

In order to remove this condition, we consider a suitable partition of the interval $[0, T]$. Let $\tilde{T}>0$ satisfy (4.7) and $0<\tilde{T}<2 \tilde{T}<\cdots<K \tilde{T}=T$ for some $K \in \mathbb{N}$. Fix $k \in\{1, \ldots, K\}$. We will study the processes $u^{n}, n \in \mathbb{N}$, on the interval $[(k-1) \tilde{T}, k \tilde{T}]$ and find an estimate similar to (4.8). Each $u^{n}, n \in \mathbb{N}$, is the unique mild solution to the corresponding linear equation

$$
\begin{aligned}
\mathrm{d} u^{n} & =\left[\mathcal{A} u^{n}+F\left(u^{n-1}\right)\right] \mathrm{d} t+\sigma\left(u^{n-1}\right) \mathrm{d} W, \quad x \in \mathbb{T}^{N}, t \in(0, T), \\
u(0) & =u_{0} .
\end{aligned}
$$

Let $v\left(t, s, ; u_{0}\right), t \geq s \geq 0$, be the mild solution of this problem with the initial condition $u_{0}$ given at time $s$. It follows from the uniqueness that for arbitrary $t \geq r \geq s \geq 0$

$$
v\left(t, r ; v\left(r, s ; u_{0}\right)\right)=v\left(t, s ; u_{0}\right) \quad \mathbb{P} \text {-a.s. }
$$

and therefore we can write

$$
\begin{aligned}
u^{n}(t)= & \mathcal{S}_{p}(t-(k-1) \tilde{T}) u^{n}((k-1) \tilde{T})+\int_{(k-1) \tilde{T}}^{t} \mathcal{S}_{p}(t-s) F\left(u^{n-1}(s)\right) \mathrm{d} s \\
& +\int_{(k-1) \tilde{T}}^{t} \mathcal{S}_{p}(t-s) \sigma\left(u^{n-1}(s)\right) \mathrm{d} W(s), \quad t \in[(k-1) \tilde{T}, T] .
\end{aligned}
$$

Following the same approach as above we obtain

$$
\mathbb{E} \sup _{(k-1) \tilde{T} \leq t \leq k \tilde{T}}\left\|u^{n}(t)\right\|_{W^{1, p}\left(\mathbb{T}^{N}\right)}^{q} \leq \tilde{C}_{\tilde{T}}\left(1+\mathbb{E}\left\|u^{n}((k-1) \tilde{T})\right\|_{W^{1, p}\left(\mathbb{T}^{N}\right)}^{q}\right)
$$

with a constant similar to $\tilde{C}_{T}$ in (4.8). Hence

$$
\begin{aligned}
& \mathbb{E} \sup _{(k-1) \tilde{T} \leq t \leq k \tilde{T}}\left\|u^{n}(t)\right\|_{W^{1, p}\left(\mathbb{T}^{N}\right)}^{q} \\
& \leq \tilde{C}_{\tilde{T}}\left(1+\mathbb{E} \sup _{(k-2) \tilde{T} \leq t \leq(k-1) \tilde{T}}\left\|u^{n}(t)\right\|_{W^{1, p}\left(\mathbb{T}^{N}\right)}^{q}\right) \\
& \leq \sum_{i=1}^{K}\left(\tilde{C}_{\tilde{T}}\right)^{i}+\left(\tilde{C}_{\tilde{T}}\right)^{K} \mathbb{E}\left\|u_{0}\right\|_{W^{1, p}\left(\mathbb{T}^{N}\right)}^{q} \leq \bar{C}\left(1+\mathbb{E}\left\|u_{0}\right\|_{W^{1, p}\left(\mathbb{T}^{N}\right)}^{q}\right),
\end{aligned}
$$


where the constant $\bar{C}$ is independent of $k$ and $n$. Finally, the estimate (4.6) follows:

$$
\begin{aligned}
& \mathbb{E} \sup _{0 \leq t \leq T}\left\|u^{n}(t)\right\|_{W^{1, p}\left(\mathbb{T}^{N}\right)}^{q}=\mathbb{E} \max _{k=1, \ldots, K} \sup _{(k-1) \tilde{T} \leq t \leq k \tilde{T}}\left\|u^{n}(t)\right\|_{W^{1, p}\left(\mathbb{T}^{N}\right)}^{q} \\
& \leq \sum_{k=1}^{K} \mathbb{E} \sup _{(k-1) \tilde{T} \leq t \leq k \tilde{T}}\left\|u^{n}(t)\right\|_{W^{1, p}\left(\mathbb{T}^{N}\right)}^{q} \leq K \bar{C}\left(1+\mathbb{E}\left\|u_{0}\right\|_{W^{1, p}\left(\mathbb{T}^{N}\right)}^{q}\right) .
\end{aligned}
$$

We have now all in hand to deduce that the sequence $\left\{u^{n} ; n \in \mathbb{N}\right\}$ is bounded in

$$
L^{q}\left(\Omega ; L^{\infty}\left(0, T ; W^{1, p}\left(\mathbb{T}^{N}\right)\right)\right)
$$

and therefore has a weak-star convergent subsequence. Since any norm is weak-star lower semicontinuous we get the estimate (4.5) for the limit process $u$. Moreover, since the stochastic convolution has a continuous modification according to [3, Corollary 3.5], the proof is complete.

Proof of regularity in higher order Sobolev spaces (order greater than 1) is more complicated as the norm of a superposition does not, in general, grow linearly with the norm of the inner function (cf. Proposition 3.1, Corollary 3.2, Remark 3.3).

Proposition 4.3. (Estimate in $W^{m, p}\left(\mathbb{T}^{N}\right)$ ) Let $p \in[2, \infty), q \in(2, \infty), m \in \mathbb{N}$, $m \geq 2$. Assume that $u_{0} \in L^{q}\left(\Omega ; W^{m, p}\left(\mathbb{T}^{N}\right)\right) \cap L^{m q}\left(\Omega ; W^{1, m p}\left(\mathbb{T}^{N}\right)\right)$ and

$$
f_{\alpha} \in C^{m}(\mathbb{R}) \cap C^{2 l-1}(\mathbb{R}), \quad|\alpha| \leq 2 l-1 ; \quad \sigma_{i} \in C^{m}\left(\mathbb{T}^{N} \times \mathbb{R}\right), \quad i=1, \ldots, d,
$$

have bounded derivatives up to order $m$. Then the mild solution of (1.1) belongs to

$$
L^{q}\left(\Omega ; C\left([0, T] ; W^{m, p}\left(\mathbb{T}^{N}\right)\right)\right)
$$

and the following estimate holds true

$$
\mathbb{E} \sup _{0 \leq t \leq T}\|u(t)\|_{W^{m, p}\left(\mathbb{T}^{N}\right)}^{q} \leq C\left(1+\mathbb{E}\left\|u_{0}\right\|_{W^{m, p}\left(\mathbb{T}^{N}\right)}^{q}+\mathbb{E}\left\|u_{0}\right\|_{W^{1, m p}\left(\mathbb{T}^{N}\right)}^{m q}\right) .
$$

Proof. First, we intend to prove the following estimate for the Picard iterations

$$
\mathbb{E} \sup _{0 \leq t \leq T}\left\|u^{n}(t)\right\|_{W^{m, p}\left(\mathbb{T}^{N}\right)}^{q} \leq C\left(1+\mathbb{E}\left\|u_{0}\right\|_{W^{m, p}\left(\mathbb{T}^{N}\right)}^{q}+\mathbb{E}\left\|u_{0}\right\|_{W^{1, m p}\left(\mathbb{T}^{N}\right)}^{m q}\right),
$$

with a constant independent of $n$. By induction on $n$, assume that the hypothesis is satisfied for $u^{n-1}$ and compute the estimate for $u^{n}$. The following arguments and calculations are mostly similar to those in Proposition 4.2. Recall that according to (4.6), we have

$$
\mathbb{E} \sup _{0 \leq t \leq T}\left\|u^{n}(t)\right\|_{W^{1, m p}\left(\mathbb{T}^{N}\right)}^{m q} \leq C\left(1+\mathbb{E}\left\|u_{0}\right\|_{W^{1, m p}\left(\mathbb{T}^{N}\right)}^{m q}\right), \quad \forall n \in \mathbb{N} .
$$

Let us consider the restrictions of the operators $\mathcal{S}_{p}(t), t \geq 0$, to the Sobolev space $W^{m, p}\left(\mathbb{T}^{N}\right)$ and denote them by $\mathcal{S}_{m, p}(t), t \geq 0$. By [2, Theorem V.2.1.3], 
we obtain a strongly continuous semigroup on $W^{m, p}\left(\mathbb{T}^{N}\right)$ generated by part of $\mathcal{A}_{p}$ in $W^{m, p}\left(\mathbb{T}^{N}\right)$. We denote the generator by $\mathcal{A}_{m, p}$. It follows

$$
\begin{aligned}
\mathbb{E} \sup _{0 \leq t \leq T}\left\|\mathcal{S}_{p}(t) u_{0}\right\|_{W^{m, p}\left(\mathbb{T}^{N}\right)}^{q} & =\mathbb{E} \sup _{0 \leq t \leq T}\left\|\mathcal{S}_{m, p}(t) u_{0}\right\|_{W^{m, p}\left(\mathbb{T}^{N}\right)}^{q} \\
& \leq C \mathbb{E}\left\|u_{0}\right\|_{W^{m, p}\left(\mathbb{T}^{N}\right)}^{q} .
\end{aligned}
$$

As above, we employ the following bounded operator: let $\delta=\frac{2 l-1}{2 l}$

$$
\begin{aligned}
\mathcal{B}_{m, p}: W^{m, p}\left(\mathbb{T}^{N} ; \mathbb{R}^{\eta}\right) & \longrightarrow W^{m, p}\left(\mathbb{T}^{N}\right) \\
\left\{z_{\alpha}\right\}_{\substack{|\alpha| \leq 2 l-1 \\
a_{\alpha} \neq 0}} & \longrightarrow\left(-\mathcal{A}_{p}\right)^{-\delta} \sum_{\substack{|\alpha| \leq 2 l-1 \\
a_{\alpha} \neq 0}} a_{\alpha} \mathrm{D}^{\alpha} z_{\alpha},
\end{aligned}
$$

so

$$
\begin{aligned}
& \mathbb{E} \sup _{0 \leq t \leq T}\left\|\int_{0}^{t} \mathcal{S}_{p}(t-s) F\left(u^{n-1}(s)\right) \mathrm{d} s\right\|_{W^{m, p}\left(\mathbb{T}^{N}\right)}^{q} \\
& \leq \mathbb{E} \sup _{0 \leq t \leq T}\left(\int_{0}^{t}\left\|\left(-\mathcal{A}_{m, p}\right)^{\delta} \mathcal{S}_{m, p}(t-s) \mathcal{B}_{m, p} f\left(u^{n-1}(s)\right)\right\|_{W^{m, p}\left(\mathbb{T}^{N}\right)} \mathrm{d} s\right)^{q} \\
& \leq C \mathbb{E} \sup _{0 \leq t \leq T}\left(\int_{0}^{t} \frac{1}{(t-s)^{\delta}}\left\|f\left(u^{n-1}(s)\right)\right\|_{W^{m, p}\left(\mathbb{T}^{N}\right)} \mathrm{d} s\right)^{q} \\
& \leq C T^{q(1-\delta)} \mathbb{E} \sup _{0 \leq t \leq T}\left\|f\left(u^{n-1}(t)\right)\right\|_{W^{m, p}\left(\mathbb{T}^{N}\right)}^{q} .
\end{aligned}
$$

And for the stochastic term, $z \in W^{m, p}\left(\mathbb{T}^{N}\right)$,

$$
\begin{aligned}
\|\sigma(z)\|_{\gamma\left(\mathfrak{U} ; W^{m, p}\left(\mathbb{T}^{N}\right)\right)}^{q} & =\left(\mathbb{E}\left\|\sum_{i=1}^{d} \gamma_{i} \sigma_{i}(\cdot, z(\cdot))\right\|_{W^{m, p}\left(\mathbb{T}^{N}\right)}^{2}\right)^{\frac{q}{2}} \\
& \leq C\left(\mathbb{E}\left\|\sum_{i=1}^{d} \gamma_{i}\left(-\mathcal{A}_{p}\right)^{\frac{m}{2 l}} \sigma_{i}(\cdot, z(\cdot))\right\|_{L^{p}\left(\mathbb{T}^{N}\right)}^{p}\right)^{\frac{q}{p}} \\
& =C\left(\int_{\mathbb{T}^{N}}\left(\sum_{i=1}^{d}\left|\left(-\mathcal{A}_{p}\right)^{\frac{m}{2 l}} \sigma_{i}(y, z(y))\right|^{2}\right)^{\frac{p}{2}} \mathrm{~d} y\right)^{\frac{q}{p}} \\
& \leq C \sum_{i=1}^{d}\left\|\sigma_{i}(\cdot, z(\cdot))\right\|_{W^{m, p}\left(\mathbb{T}^{N}\right)}^{q}
\end{aligned}
$$


hence

$$
\begin{aligned}
& \mathbb{E} \sup _{0 \leq t \leq T}\left\|\int_{0}^{t} \mathcal{S}_{p}(t-s) \sigma\left(u^{n-1}(s)\right) \mathrm{d} W(s)\right\|_{W^{m, p}\left(\mathbb{T}^{N}\right)}^{q} \\
& \leq C T^{\frac{q}{2}-1} \mathbb{E} \int_{0}^{T}\left\|\sigma\left(u^{n-1}(s)\right)\right\|_{\gamma\left(\mathfrak{U} ; W^{m, p}\left(\mathbb{T}^{N}\right)\right)}^{q} \mathrm{~d} s \\
& \leq C T^{\frac{q}{2}} \sum_{i=1}^{d} \mathbb{E} \sup _{0 \leq t \leq T}\left\|\sigma_{i}\left(\cdot, u^{n-1}(t, \cdot)\right)\right\|_{W^{m, p}\left(\mathbb{T}^{N}\right)}^{q} .
\end{aligned}
$$

We conclude

$$
\begin{aligned}
\mathbb{E} \sup _{0 \leq t \leq T}\left\|u^{n}(t)\right\|_{W^{m, p}\left(\mathbb{T}^{N}\right)}^{q} \leq & C \mathbb{E}\left\|u_{0}\right\|_{W^{m, p}\left(\mathbb{T}^{N}\right)}^{q} \\
& +C T^{q(1-\delta)} \mathbb{E} \sup _{0 \leq t \leq T}\left\|f\left(u^{n-1}(t)\right)\right\|_{W^{m, p}\left(\mathbb{T}^{N}\right)}^{q} \\
& +C T^{\frac{q}{2}} \sum_{i=1}^{d} \mathbb{E} \sup _{0 \leq t \leq T}\left\|\sigma_{i}\left(\cdot, u^{n-1}(t, \cdot)\right)\right\|_{W^{m, p}\left(\mathbb{T}^{N}\right)}^{q} .
\end{aligned}
$$

Applying Proposition 3.1, Corollary 3.2 and (4.11) we obtain

$$
\begin{aligned}
\mathbb{E} \sup _{0 \leq t \leq T}\left\|u^{n}(t)\right\|_{W^{m, p}\left(\mathbb{T}^{N}\right)}^{q} \leq C \mathbb{E}\left\|u_{0}\right\|_{W^{m, p}\left(\mathbb{T}^{N}\right)}^{q}+C\left(T^{q(1-\delta)}+T^{\frac{q}{2}}\right) \\
\quad \times\left(1+\mathbb{E} \sup _{0 \leq t \leq T}\left\|u^{n-1}(t)\right\|_{W^{1, m p}\left(\mathbb{T}^{N}\right)}^{m q}+\mathbb{E} \sup _{0 \leq t \leq T}\left\|u^{n-1}(t)\right\|_{W^{m, p}\left(\mathbb{T}^{N}\right)}^{q}\right) \\
\leq C \mathbb{E}\left\|u_{0}\right\|_{W^{m, p}\left(\mathbb{T}^{N}\right)}^{q}+C\left(T^{q(1-\delta)}+T^{\frac{q}{2}}\right) \\
\quad \times\left(1+\mathbb{E}\left\|u_{0}\right\|_{W^{1, m p}\left(\mathbb{T}^{N}\right)}^{m q}+\mathbb{E} \sup _{0 \leq t \leq T}\left\|u^{n-1}(t)\right\|_{W^{m, p}\left(\mathbb{T}^{N}\right)}^{q}\right) .
\end{aligned}
$$

Let $T$ satisfy the following condition

$$
C_{T}=C\left(T^{q(1-\delta)}+T^{\frac{q}{2}}\right)<1
$$

and define $K_{n}=\mathbb{E} \sup _{0 \leq t \leq T}\left\|u^{n}(t)\right\|_{W^{m, p}\left(\mathbb{T}^{N}\right)}^{q}, n \in \mathbb{N}_{0}, L_{0}=\mathbb{E}\left\|u_{0}\right\|_{W^{1, m p}\left(\mathbb{T}^{N}\right)}^{m q}$. Then we have

$$
K_{n} \leq C \mathbb{E}\left\|u_{0}\right\|_{W^{m, p}\left(\mathbb{T}^{N}\right)}^{q}+C_{T}\left(1+L_{0}+K_{n-1}\right)
$$

hence inductively in $n$

$$
\mathbb{E} \sup _{0 \leq t \leq T}\left\|u^{n}(t)\right\|_{W^{m, p}\left(\mathbb{T}^{N}\right)}^{q} \leq \tilde{C}_{T}\left(1+\mathbb{E}\left\|u_{0}\right\|_{W^{m, p}\left(\mathbb{T}^{N}\right)}^{q}+\mathbb{E}\left\|u_{0}\right\|_{W^{1, m p}\left(\mathbb{T}^{N}\right)}^{m q}\right),
$$

where the constant does not depend on $n$. Therefore (4.10) follows under the additional hypothesis upon $T$. However, this condition can be removed by the same approach as in Proposition 4.2. 
Similarly to Proposition 4.2 we deduce that the sequence $\left\{u^{n} ; n \in \mathbb{N}\right\}$ is bounded in

$$
L^{q}\left(\Omega ; L^{\infty}\left(0, T ; W^{m, p}\left(\mathbb{T}^{N}\right)\right)\right)
$$

and therefore (4.9) holds true. Existence of a continuous modification follows again from [3, Corollary 3.5].

Proof of Theorem 2.1. If $m=1$ the proof is an immediate consequence of Propositions 4.1 and 4.2. The case $m \geq 2$ follows from Propositions 4.1, 4.2 and 4.3 .

Proof of Corollary 2.2. Let $m=k+1$. According to Theorem 2.1 there exists a solution of (1.1) which belongs to

$$
L^{q}\left(\Omega ; C\left([0, T] ; W^{m, p}\left(\mathbb{T}^{N}\right)\right)\right), \quad \forall p \in[2, \infty) .
$$

If $p>N$, then according to the Sobolev embedding theorem, the space $W^{m, p}\left(\mathbb{T}^{N}\right)$ is continuously embedded in $C^{k, \lambda}\left(\mathbb{T}^{N}\right)$ for $\lambda \in(0,1-N / p)$. Hence the assertion follows.

\section{Acknowledgments}

The author is indebted to Arnaud Debussche and Jan Seidler for valuable consultations and comments.

\section{References}

[1] Amann, H., Hieber, M., Simonett, G.: Bounded $H_{\infty}$-calculus for elliptic operators. Differ. Integr. Equ. 7, 613-653 (1994)

[2] Amann, H.: Linear and Quasilinear Parabolic Problems. Birkhäuser, Basel (1995)

[3] Brzeźniak, Z.: On stochastic convolution in Banach spaces and applications. Stoch. Stoch. Rep. 61, 245-295 (1997)

[4] Brzeźniak, Z.: Stochastic partial differential equations in M-type 2 Banach spaces. Potential Anal. 4, 1-45 (1995)

[5] Da Prato, G., Zabczyk, J.: Stochastic equations in infinite dimensions. In: Encyclopedia Math. Appl., vol. 44. Cambridge University Press, Cambridge (1992)

[6] Flandoli, F.: Dirichlet boundary value problem for stochastic parabolic equations: compatibility relations and regularity of solutions. Stoch. Stoch. Rep. 29(3), 331-357 (1990)

[7] Friedman, A.: Partial Differential Equations of Parabolic Type. Prentice-Hall, Englewood Cliffs (1964)

[8] Grunau, H.C., von Wahl, W.: Regularity of weak solutions of semilinear parabolic systems of arbitrary order. J. Anal. Math. 62, 307-322 (1994) 
[9] Gyöngy, I., Rovira, C.: On $L^{p}$-solutions of semilinear stochastic partial differential equations. Stoch. Process. Appl. 90, 83-108 (2000)

[10] Hofmanová, M.: Degenerate parabolic stochastic partial differential equations. To appear

[11] Krylov, N.V.: A $W_{2}^{n}$-theory of the Dirichlet problem for SPDEs in general smooth domains. Probab. Theory Relat. Fields 98(3), 389-421 (1994)

[12] Krylov, N.V., Rozovskii, B.L.: On the Cauchy problem for linear stochastic partial differential equations. Izv. Akad. Nauk. SSSR Ser. Mat. 41(6), 1329-1347 (1977); English transl. Math. USSR Izv. 11 (1977)

[13] Krylov, N.V., Rozovskii, B.L.: Stochastic evolution equations. Itogi Nauki i Tekhniki. Ser. Sovrem. Probl. Mat. 14, 71-146 (1979). VINITI, Moscow; English transl. J. Sov. Math. 16(4), 1233-1277 (1981)

[14] Ladyzhenskaya, O.A., Solonnikov, V.A., Ural'ceva, N.N.: Linear and quasilinear equations of parabolic type. Translations of Mathematical Monographs, vol. 23. Am. Math. Soc., Providence (1968)

[15] Lieberman, G.M.: Second Order Parabolic Differential Equations. World Scientific, River Edge (1996)

[16] Ondreját, M.: Uniqueness for stochastic evolution equations in Banach spaces. Dissert. Math. 426, 1-63 (2004)

[17] Pazy, A.: Semigroups of Linear Operators and Applications to Partial Differential Equation. Applied Mathematical Sciences, vol. 44. Springer, New York (1983)

[18] Runst, T., Sickel, W.: Sobolev spaces of fractional order, Nemytskij operators, and nonlinear partial differential equations. de Gruyter Series in Nonlinear Analysis and Applications, vol. 3. Walter de Gruyter, Berlin (1996)

[19] von Wahl, W.: Extension of a result of Ladyzhenskaya and Ural'ceva concerning second order parabolic equations of arbitrary order. Ann. Pol. Math. 41, 63$72(1983)$

[20] Yagi, A.: Abstract Parabolic Evolution Equations and their Applications. Springer, Berlin (2010)

[21] Zhang, X.: Smooth solutions of non-linear stochastic partial differential equations driven by multiplicative noises. Sci. China Math. 53, 2949-2972 (2010)

[22] Zhang, X.: Regularities for semilinear stochastic partial differential equations. J. Funct. Anal. 249, 454-476 (2007)

Martina Hofmanová

Department of Mathematical Analysis

Faculty of Mathematics and Physics

Charles University

Sokolovská 83

18675 Praha 8

Czech Republic 
Institute of Information Theory and Automation of the ASCR

Pod Vodárenskou věží 4

18208 Praha 8

Czech Republic

IRMAR

ENS Cachan Bretagne

CNRS

UEB av. Robert Schuman

35170 Bruz

France

e-mail: martina.hofmanova@bretagne.ens-cachan.fr

Received: 28 November 2011.

Accepted: 28 May 2012. 